\title{
Development and Plasticity in Sensory Thalamus and Cortex
}




\section{Development and Plasticity in Sensory Thalamus and Cortex}

Edited by

Reha Erzurumlu

LSU Health Sciences Center

New Orleans, LA, USA

\section{William Guido}

LSU Health Sciences Center

New Orleans, LA, USA

Zoltán Molnár

University of Oxford

Oxford, UK

黑 Springer 
Reha Erzurumlu

LSU Health Sciences Center

Department of Cell

Biology and Anatomy

New Orleans, LA
William Guido

LSU Health Sciences Center

Department of Cell

Biology and Anatomy

New Orleans, LA

\author{
Zoltan Molnar \\ University of Oxford \\ Department of Anatomy and \\ Genetics \\ Oxford, UK
}

Library of Congress Control Number: 2006920147

ISBN-10: 0-387-31798-8

ISBN-13: 978-0387-31798-4

Printed on acid-free paper.

(C) 2006 Springer Science+Business Media, LLC

All rights reserved. This work may not be translated or copied in whole or in part without the written permission of the publisher (Springer Science+Business Media, LLC, 233 Spring Street, New York, NY 10013, USA), except for brief excerpts in connection with reviews or scholarly analysis. Use in connection with any form of information storage and retrieval, electronic adaptation, computer software, or by similar or dissimilar methodology now known or hereafter developed is forbidden.

The use in this publication of trade names, trademarks, service marks, and similar terms, even if they are not identified as such, is not to be taken as an expression of opinion as to whether or not they are subject to proprietary rights.

Printed in Singapore (KYO)

988765543321

springer.com 


\section{In Memory of Ary S. Ramoa, MD, MSc, Ph.D.}

Dr. Ary S. Ramoa, neuroscientist, scholar, teacher, physician, husband, father, and friend to many, passed away on July 1, 2005 from complications of Crohn's disease. Ary was one of the speakers at the satellite symposium. We are all deeply saddened by his loss and this book is dedicated to his memory and contribution to the field.

Ary was born in Rio de Janeiro, Brazil on December 21, 1953. In 1979, he received his M.D. from the State University of Rio de Janeiro. Having already uncovered his passion for research, he continued his training

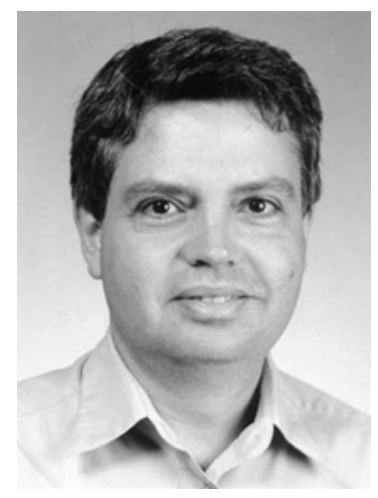
in the laboratory of Dr. Carlos Eduardo Rocha-Miranda at the Federal University of Rio de Janiero. Here, he made some of his first observations of the structure and function of the visual system related to the specific contribution of each eye, a paradigm which later became a particularly successful tool in his own research laboratory. In 1981, Ary received his M.Sc. Degree in Biophysics and moved, with the help of an NIH Fogarty Fellowship, to the United States to earn his Ph.D. His doctoral research at the University of California at Berkeley with Dr. Ralph Freeman continued his examination of the visual system. This was a particularly productive experience for him, in which he not only examined response properties of visual thalamic and cortical neurons, but also was exposed to the tools of developmental manipulation of visual experience and pharmacological manipulation. After receiving his Ph.D. in Physiological Optics in 1986, he pursued postdoctoral training with Dr. Carla Shatz at Stanford University, Dr. Mriganka Sur at the Massachusetts Institute of Technology, and Drs. Pasko Rakic and David McCormick at Yale University. In these positions, he acquired the necessary skills to merge techniques of experiential manipulation, neuropharmacology and electrophysiology to assess the basic mechanisms of visual development. By the time he joined the faculty of the Department of Anatomy and Neurobiology at Virginia Commonwealth University School of Medicine in 1993, Ary had not only studied with some of the most influential visual and developmental neuroscientists in the world, but also had devised a sophisticated, novel and effective approach to his primary research interest. 
Supported by multiple NIH and foundation grant awards, Ary's independent work began by addressing the effect of amblyopia on neural development and plasticity. Amblyopia is a neurological disorder characterized by reduced vision in one eye which afflicts millions of people worldwide. Using an animal model of amblyopia, he pioneered the in vivo use of viral-mediated gene transfer and antisense techniques in combination with monocular deprivation to discover critical factors involved in loss of visual function. These studies also led to the next logical step: the examination of the factors that regulate recovery of visual cortical function. This work, ongoing at the time of his final illness, showed that different mechanisms control the loss and the recovery phases of visual plasticity. Ary also recently developed another line of research: investigations into the relationship of visual cortical plasticity to Fetal Alcohol Syndrome, a leading cause of mental retardation. Using the animal model of Fetal Alcohol Syndrome which he and his collaborators had devised, early alcohol exposure was documented to reduce neural plasticity necessary for the normal development of visual cortical processing. Collectively, Ary's findings, which have won him national and international attention, have contributed significantly to the fundamental understanding of how sensory experience relates to loss and recovery of brain function. Ultimately, these efforts will provide the conceptual substrate upon which therapeutic strategies can be devised to reduce the impact of sensory deprivation or loss and to enhance the neuroplastic potential for rehabilitation in diverse neurological disorders.

With his devotion not only to research, but also to his friends, family and colleagues, Ary leaves behind a vast number of people whose lives were touched by his presence. He was an outstanding role model for his students and post-docs and provided excellent council, spirit and humor to his fellow researchers and colleagues. Consequently, he received the Virginia Commonwealth University Research Achievement Award in 2004 and in 2005. Ary will also be remembered for his intellect, enthusiasm, perseverance, smile, kind-heartedness, dedication to the advancement of science and love for his family and friends. He was respected worldwide, esteemed by those who knew him and loved dearly by those closest to him. He will truly be missed.

M. Alex Meredith

Virginia Commonwealth University

Richmond, Virginia 


\section{Contents}

$\begin{array}{ll}\text { Preface } & \text { ix }\end{array}$

Contributors $\quad$ xiii

1 Pioneer Neurons and Interneurons in the Developing Subplate: Molecular Markers, Cell Birthdays, and Neurotransmitters

Robert F. Hevner and Nada Zecevic

2 Dual Roles of Transcription Factors in Forebrain Morphogenesis and Development of Axonal Pathways....... Thomas Pratt and David J. Price

3 Subcortical and Neocortical Guidance of Area-specific Thalamic Innervation.

Tomomi Shimogori and Elizabeth A. Grove

4 The Earliest Thalamocortical Interactions.

Zoltán Molnár, Guillermina López-Bendito, Daniel Blakey, Alexander Thompson, and Shuji Higashi

5 Molecular Basis for the Formation of Lamina-Specific Thalamocortical Projection

Nobuhiko Yamamoto, Makoto Takemoto, Yuki Hattori, and Kenji Hanamura

6 Role of Citron $\mathrm{K}$ in the Development of Cerebral Cortex..... 92 Patrizia Muzzi, Paola Camera, Ferdinando Di Cunto, and Alessandro Vercelli

7 The Absence of Layer 4 Dramatically Alters Cortical Development in Ferret Somatosensory Cortex.

Debra F. McLaughlin, Sylvie Poluch, Beata Jablonska, and Sharon L. Juliano

8 Influence of Thalamocortical Activity on Sensory Cortical Development and Plasticity

Sarah L. Pallas, Mei Xu, and Khaleel A. Razak 
9 Pathways to Barrel Development.......................... 138 Mark W Barnett, R. F. Watson, and Peter C. Kind

10 Patterning of the Somatosensory Maps with NMDA Receptors.

Reha S. Erzurumlu and Takuji Iwasato

11 Presynaptic Mechanisms Controlling Axon Terminal Remodeling in the Thalamocortical and Retinogeniculate Systems....

Alexandra Rebsam and Patricia Gaspar

12 Cellular Mechanisms Underlying the Remodeling of Retinogeniculate Connections

William Guido

13 A Model for Synaptic Refinement in Visual Thalamus

Bryan M. Hooks and Chinfei Chen

14 Making and Breaking Eye-specific Projections to the Lateral Geniculate Nucleus....

Andrew D. Huberman and Barbara Chapman

15 LTD as a Mechanism for Map Plasticity in Rat

Barrel Cortex.

Kevin J. Bender, Suvarna Deshmukh, and Daniel E. Feldman

16 High-Field (9.4T) Magnetic Resonance Imaging in Squirrel Monkey

Aimee J. Nelson, Cheryl A. Cheney, Yin-Ching Iris Chen, Guangping Dai, Robert P. Marini, Graham C. Grindlay, Yumiko Ishizawa, and Christopher I. Moore

Index 


\section{Preface}

The idea of this book was conceived during a satellite meeting of the Society for Neuroscience entitled "Development and Plasticity in Sensory Thalamus and Cortex in 2003. The meeting was hosted by the LSU Health Sciences Center, New Orleans and organized by Reha Erzurumlu, William Guido and Zoltán Molnár. That occasion brought together systems and molecular approaches on various key issues on thalamocortical development and plasticity. The sheer volume and concentration of presentations specifically dedicated to these issues distinguished this satellite meeting from other cortical development conferences. The meeting was greeted with great enthusiasm and we all felt that more should follow. When the idea of the book was discussed the majority of the participants decided to contribute with a timely review on their specific field of expertise. Additional leading experts, who were not attending the original New Orleans Meeting, were also invited to contribute with relevant topics.

The 16 chapters each presents topics central to thalamocortical development and plasticity ranging from the establishment of thalamic sensory innervation to imaging cortical function in primates. Hevner and Zecevic start by introducing the concepts of forebrain patterning and axon guidance as well as discussing recent discoveries on the changes in intra- and extracortical circuits during development. Recent progress uncovered some of the mechanisms involved in thalamic axon deployment through the embryonic forebrain to the cortex and the subsequent intracortical guidance mechanisms. The largely transient cell population forming the subplate is central to this process. Understanding of various subplate cell types and their neurochemical properties is driven by excellent transgenic mouse model systems, some of which are discussed here.

Pratt and Price describe the mechanisms which allow thalamic axons to cross several diencephalic and telencaphalic boundaries as they approach the cortex. These compartments are patterned by overlapping gene expression gradients, and as our knowledge on forebrain patterning increases, so does the understanding of the thalamocortical guidance mechanisms. Using in vitro approaches and genetic manipulation it has been possible to dissect out the role that individual transcription factors have on thalamocortical axon guidance and the indirect affects on forebrain patterning.

Shimogori and Grove illustrate the power of transgenic mouse models and in vivo electroporation in the context of understanding subcortical 
and neocortical guidance mechanisms leading to area-specific thalamocortical innervation. These issues are closely related to the key questions in cortical arealization, as altering the expression of a single gene during early cortical development can not only change the fate of the cortex, but also change the destination of thalamocortical fibres. Surprisingly, the majority of thalamocortical rearrangement in such paradigms occurs at the cortical level. Specifically the early mis-expression of FGF-8 does not change the subcortical trajectories of the thalamocortical projections, but has a drastic effect on the intracortical targeting. This suggests that the pathfinding of the thalamic projections to the subplate relies on different signals, than the subsequent intracortical guidance.

The mechanism of this intracortical rearrangement is not clear, but it has been observed in other model systems (Chapter 11 Rebsam and Gaspar; Chapter 4 Molnár and colleagues). Chapter 4 examines the changes in thalamocortical relations in paradigms when the sensory periphery, the extent of the cortical sheet or the trajectories of the thalamocortical projections are altered. All of these result in large scale area and lamina-specific rearrangements of the thalamocortical projections within the cerebral cortex, which is in contrast to previous suggestions that thalamocortical projections are specified from the time they leave the lower tier of the cortical plate.

Yamamoto and colleagues review the molecular mechanisms involved in lamina-specific thalamocortical targeting. Although thalamic projections innervate every single cortical layer, their major target is layer 4 . The challenge is to identify the dominant repulsive and attractive cues in the cortex at the time when cortical lamination and cellular differentiation are incomplete. The tools of modern molecular biology enabled spectacular progress in this field, providing numerous mutants, to test our current ideas on cortical development.

In Chapter 6 the role of Citron $\mathrm{K}$ and the effects of its deletion on cortical development are analyzed by Vercelli and colleagues. They show that the barrelfield can develop also in a microencephalic brain, and still responds to peripheral manipulations. Citron $\mathrm{K}$ plays a fundamental role in cytokinesis and dendritic development, and its deletion causes neuronal depletion resulting in reduced cortical mantle size which reflects in shrinkage of the barrelfield, and in altered distribution of cortical interneurons.

Juliano and colleagues examine the consequences of a reduction in or absence of layer 4 cells on thalamocortical development and cortical circuit formation. They provide further evidence of a layer 4-specific stop signal.

In chapters 9, 10 and 11 the functional aspects of early thalamocortical interactions are discussed. The first synaptic transmission between thalamic fibres and subplate cells start shortly after the thalamic projections arrive to the cortical subplate. Thalamic projections are able to elicit dynamic patterns of cortical activities which are believed to determine the area and lamina-specific thalamic ingrowth and influence on the cortical circuit formation and plasticity. Due to the 
pioneering work of Van der Loos and Woolsey, the barrel cortex of the primary somatosensory cortex became a very useful model system in understanding the causal relationships between the periphery related thalamocortical fibre patterning and the formation of the characteristic cytoarchitectonic features, the barrels. Kind, Erzurumlu, Gaspar and their colleagues review the progress emerging from various transgenic mouse lines used to understand the role of pre- and post-synaptic signaling machinery involvement in barrel formation. Disruption of the pre-synaptic release mechanisms prevents thalamocortical axons from forming the periphery related pattern, whereas disruption of the postsynaptic receptor mediated signal transduction mechanisms on cortical cells inhibits the formation of the characteristic cytoarchitectonic barrel pattern. These models increased our understanding of the molecular and cellular mechanisms of thalamocortical axon pathfinding and arbor formation, remodeling and plasticity. Unfortunately our current genetic models do not manipulate these signaling mechanisms selectively and specifically in the thalamocortical synapse; the synapses of the sensory organs, thalamus, and cortex are also affected. The future challenge will be to understand the contributions at each specific level.

The barrel field also provides an excellent system for understanding plasticity. In Chapter 15 Feldman and colleagues describe long term depression mechanisms of sensory map plasticity in the barrel cortex. Both the activity level and activity pattern are instrumental in shaping the thalamocortical network during development. Pallas and colleagues changed the pattern of activity delivered through thalamocortical pathways but not the thalamocortical connectivity itself, in their re-wired brain models. They demonstrated the existence of modality specific activity, which plays an important role in shaping cortical circuitry.

In Chapters 12 to 14 Guido, Hooks and Chen, Huberman and Chapman review recent developments affecting our understanding of the cellular and molecular interactions between retinal projections and the lateral geniculate nucleus of the thalamus. They also propose new rules for the establishment and modification of this pathway. Functional cortical circuits cannot be appreciated without thalamocortical circuits and cortical development cannot be considered without thalamocortical pathway development. But this in turn is influenced by the way that the connectivity between sensory organs and thalamus develops. To this end, the eye-specific termination pattern of retinal ganglion cells is examined in various paradigms. Here the retino-geniculate projection is always examined in the context of the entire sensory pathway, considering the functional implications of alterations in the system. Moore and colleagues further investigate the functional consequences in an fMRI study presented in Chapter 16.

This book is not intended to be exhaustive. We were, for obvious reasons, unable to cover all aspects of thalamocortical development. Since the corticofugal projections dominate the input to the thalamus, the recent progress in the illuminating the mechanisms of corticofugal development should have been reviewed. Transgenic models with 
reporter gene expression and in vivo electroporation have led to interesting developments in this particular field. Also, perhaps we would have liked to include human imaging studies with possible clinical implications (including the thalamocortical contribution to syndromes such as phantom limb, autism, schizophrenia and synesthesia). Nevertheless, we hope that the book reflects the tremendous enthusiasm and the atmosphere that surrounded the meeting at New Orleans. We are especially grateful to Dr. Nicolas Bazan, Director of the LSU Neuroscience Center. His generosity, hospitality and intellectual support and contributions to the symposium paved the way to this work.

We dedicate this book to Ary Ramoa, who passed away from complications of Crohns disease in July 2005. Ary was a contributor to the symposium. He was a dear friend and colleague of many of the participants and this book is a testament to the contributions he has made. Alex Meredith was kind enough to provide a few words honoring Ary's life and scientific career.

Reha Erzurumlu, William Guido, Zoltán Molnár New Orleans and Oxford, January 2006, 


\section{Contributors}

Robert F. Hevner and Nada Zecevic Department of Pathology, University of Washington, Seattle, Washington; and Department of Neuroscience, University of Connecticut Health Center, Farmington, CT, USA.

Thomas Pratt and David J. Price Genes and Development Group, University of Edinburgh, Hugh Robson Building, George Square, Edinburgh EH8 9XD, UK.

Tomomi Shimogori and Elizabeth A. Grove Critical Period Mechanism Research Group, Institute of Physical and Chemical Research (RIKEN), Brain Science Institute, Saitama 351-0198, Japan; and Department of Neurobiology, Pharmacology and Physiology, University of Chicago, Chicago, IL, 60637, USA.

Zoltán Molnár, Guillermina López-Bendito, Daniel Blakey, Alexander Thompson, and Shuji Higashi Department of Human Anatomy and Genetics, University of Oxford, South Park Road, Oxford OX1 3QX, UK, Institute de Neurosciencias, CSIC-Universidad Miguel Hernandez, Campus de San Juan, 03550 San Juan de Alicante, Spain. Division of Neurophysiology, Graduate School, Kyoto Prefectural University of Medicine, Kawaranachi Hirokoji, Kyoto 602-8566 Japan.

Nobuhiko Yamamoto, Makoto Takemoto, Yuki Hattori, and Kenji Hanamura Neuroscience Laboratories, Graduate School of Frontier Biosciences, Osaka University, Suita, Osaka 565-0871, Japan.

Patrizia Muzzi, Paola Camera, Ferdinando Di Cunto, and Alessandro Vercelli Department of Anatomy, Pharmacology and Forensic Medicine, University of Torino, corso M.D'Azeglio 52, 10126 Torino, Italy.

Debra F. McLaughlin, Sylvie Poluch, Beata Jablonska, and Sharon L. Juliano Department of Anatomy, Physiology, and Genetics, and Program in Neuroscience USUHS, 4301 Jones Bridge Rd., Bethesda MD 20814.

Sarah L. Pallas, Mei Xu, and Khaleel A. Razak Department of Biology, Graduate Program in Neurobiology and Behavior, Georgia State University, Atlanta, GA, 30303 USA.

Mark W Barnett, R. F. Watson, and Peter C. Kind Center for Integrative Physiology, University of Edinburgh, EH8 9XD. 
Reha S. Erzurumlu and Takuji Iwasato Department of Cell Biology and Anatomy, LSUHSC, New Orleans, LA and PRESTO, Japan Science and Technology Agency and RIKEN brain Science Institute, Saitama, Japan.

Alexandra Rebsam and Patricia Gaspar INSERM U 616, Universite de Paris VI, Hopital Salpetriere, 47, Blvd de 1'Hopital, 75651 Paris Cedex 13.

William Guido Louisiana State Health Sciences Center Department of Cell Biology and Anatomy and the Neuroscience Center of Excellence, New Orleans, LA, 70112.

Bryan M. Hooks and Chinfei Chen Program in Neuroscience, Harvard Medical School, and Neurobiology Program, Children's Hospital, Boston, MA 02115.

Andrew D. Huberman and Barbara Chapman Center for Neuroscience, University of California, Davis, CA 95616, USA.

Kevin J. Bender, Suvarna Deshmukh, and Daniel E. Feldman Division of Biological Sciences, University of California San Diego, La Jolla, CA, 92093-0357.

Aimee J. Nelson, Ph.D, Cheryl A. Cheney, Yin-Ching Iris Chen, PhD, Guangping Dai, PhD, Robert P. Marini, DVM, Graham C. Grindlay, MSc, Yumiko Ishizawa, MD, PhD and Christopher I. Moore, Ph.D McGovern Institute for Brain Research, Massachusetts Institute of Technology, A.A. Martinos Center for Biomedical Imaging, Massachusetts General Hospital, Division of Comparative Medicine, Massachusetts Institute of Technology, Department of Anesthesia and Critical Care, Massachusetts General Hospital, Boston MA, 02115. 